\title{
Etiology of the Neonatal Seizures: An Epidemiological Study
}

\author{
Ali Zafari', Fatemeh Pajouhandeh ${ }^{2 *}$, Mehran Arab Ahmadi ${ }^{3^{*}}$ \\ 'Department of Pediatric, Mofid Children's Hospital, Shahid Beheshti University of Medical Sciences, Tehran, Iran \\ ${ }^{2}$ Department of Pediatric, Loghman Hospital, Shahid Beheshti University of Medical Sciences, Tehran, Iran \\ ${ }^{3}$ Department of Radiology, Imam Khomeini Hospital Complex, Tehran University of Medical Sciences, Tehran, Iran
}

\begin{abstract}
Background: Neonatal seizure is a rare neurologic condition. The current study aimed at determining the etiology of neonatal seizure.

Methods: The current study evaluated the data of 100 neonates who were hospitalized at neonatal intensive care unit (NICU) during 2015-2017. A pediatric neurologist made the final diagnosis of seizure. Patients' medical records were used to review neonatal seizure variables. SPSS (version 16) was used to perform the statistical analyses.

Results: The current study enrolled 100 newborns ( $41 \%$ female) admitted to the NICU following the first episode of seizure and the body temperature of $36.8-39.2{ }^{\circ} \mathrm{C}$ (mean: $37.2^{\circ} \mathrm{C}$ ). Of 100 participants, $94(94 \%)$ had acute symptomatic seizure and $6(6 \%)$ were compatible with neonatal epilepsy syndrome criteria. According to the results, the commonest etiologies were the neonatal encephalopathy and hypoxic-ischemic encephalopathy constituting $82 \%$ of participants.

Conclusion: The consequences of acute symptomatic seizures in neonates are determined mainly by the etiology of the seizures. Seizure burden and use of anti-seizure drugs may also have some impact, but this has yet to be fully defined.

Keywords: Newborns, Seizure, Neonatal Encephalopathy
\end{abstract}

Citation: Zafari A, Pajouhandeh F, Arab Ahmadi M. Etiology of the neonatal seizures: an epidemiological study. Int Clin Neurosci J. 2019;6(4):129-132. doi:10.15171/icnj.2019.24

\section{Introduction}

To manage neonatal seizure, clinical objectives should primarily be considered as the etiology of seizure. An untreated underlying cause of seizure may lead to CNS disease, while treatments designed based on etiology of the disease can be administered following a timely detection. This, also, would lead to control the seizures themselves. The etiology of the seizure and the brain sequels grade are the major factors contributing to long-term outcomes. Several causes were identified for seizure; but a pathway of abnormal neural discharge is proven. Different clinical manifestations in seizure, ranging from subtle and nonconvulsive to dramatic generalized convulsion, depend on the abnormal discharge extent and its location in the brain. ${ }^{1}$ seizure types vary from simple febrile ones that are benign and self-limited ${ }^{2}$ to some others that cause brain damage or death. Investigation on the etiology of seizure is highlighted in the light of controversies on the efficacy of neuroimaging, electroencephalography, and some laboratory panels to manage seizure in newly experiencing children.

All neonatal seizures are "symptomatic" and have specific detectable etiology. ${ }^{3}$ Hypoxic-ischemic encephalopathy, infection/fever, developmental/neurological disorders, traumatic head injury, and metabolic disorders are the main etiologies in neonates experiencing the first episode of seizure ${ }^{4}$ that are acute and reactive and distinct from neonatal epilepsy. Although the vast majority of neonatal seizures occur as acute reactive events in response to identifiable etiologic factors, additional rare but distinct neonatal epilepsy syndromes are well recognized. These include: Benign familial neonatal epilepsy and early myoclonic encephalopathy.

Symptomatic neonatal seizures may result from a wide range of possible etiologies. ${ }^{5,6}$ These can be organized by frequency as well as relative acuity. Most etiologies can be broadly categorized as: Neonatal encephalopathy and hypoxic-ischemic encephalopathy, acquired structural brain lesions, including ischemic and hemorrhagic stroke, metabolic disturbances and CNS or systemic infections. In a prospective multicenter study of 426 consecutive neonates with seizures, hypoxic ischemic encephalopathy was the most common etiology (38\%), followed by ischemic stroke (18\%) and intracranial hemorrhage (11\%). ${ }^{7}$ Hence, there are controversies about the efficacy of routine neuroimaging and monitoring the level of blood

(C) 2019 The Author(s). This is an open access article distributed under the terms of the Creative Commons Attribution License (http:// creativecommons.org/licenses/by/4.0/), which permits unrestricted use, distribution, and reproduction in any medium, provided the original work is properly cited. 
sugar, electrolytes, and calcium in the management of first-attack or afebrile seizure children, which highlights the need for studying the etiology of neonatal seizure.

\section{Materials and Methods}

The current descriptive hospital-based study was performed in the Department of Neonatology, Loghman hospital from April 2015 to December 2017. The neonates who were hospitalized at neonatal intensive care unit (NICU) participated in the study. Neonates with a history of epilepsy, underlying diseases, e.g., metabolic disorders, and the ones who experienced seizure after birth were also included.

Demographic and clinical characteristing including age, gender, pattern of seizure, fever, history of head injury, infection, intoxication, and developmental history were collected. Serum electrolytes and blood sugar levels were also measured in all the subjects. According to manifestations, seizures were categorized as motor and non-motor in the current study.

To analyze data, descriptive statistics and hypothesis testing were employed using SPSS version 16. Relationship between variables was examined using the Chi-square test and logistic regression analysis was also employed to evaluate the strength of relationships. OR and 95\% CI were also calculated. $P<0.05$ was considered the level of significance.

\section{Results}

In the current study 100 newborns experiencing the first episode of seizure admitted to the NICU were enrolled ( $41 \%$ female). Their body temperature ranged $36.8^{\circ} \mathrm{C}$ to $39.2^{\circ} \mathrm{C}$ (mean: $37.2^{\circ} \mathrm{C}$ ). Also, 94 (94\%) subjects had acute symptomatic seizure, while the symptoms of six (6\%) were in line with the criteria of neonatal epilepsy syndrome. No significant difference was observed in the prevalence of disease types between males and females. Eight subjects (8\%) had a family history of seizure-related disorders. In addition, 37 (37\%) subjects had a history of seizure. Neonatal encephalopathy and hypoxic-ischemic encephalopathy were the most frequent (82\%) seizure etiologies in the current study. Structural brain lesions were seem in $6 \%$ of the patients.

Intracranial hemorrhage (3\%) was also a common etiology of seizure in the current study (Table 1).

\section{Discussion}

The present hospital-based, cross sectional study performed on newborns admitted to NICU following an acute episode of seizure aimed at determining the etiology and gender-specific pattern of seizure in the selected subjects. According to evidence, the commonest etiology is febrile seizure, except for the age group of 7-12 years. No significant difference was observed between males and females in terms of the prevalence of seizure etiology.

Consistent with the results of this study, in a prospective multicenter study of 426 consecutive neonates with seizures, hypoxic ischemic encephalopathy was the most common etiology (38\%), followed by ischemic stroke (18\%) and intracranial hemorrhage (11\%). ${ }^{7}$ It can be attributed to the high prevalence of neurodevelopmental disorders in patients from these age groups. One of the commonest causes of pediatric seizure is febrile seizure. ${ }^{8}$ The current study findings showed that the main seizure etiology was hypoxic-ischemic encephalopathy in the studied patients (82\%). Many neonatal seizures

Table 1. Descriptive Statistics of Patients With a First Attack of Seizure Disorder

\begin{tabular}{|c|c|c|c|c|}
\hline & Total $(n=100)$ & Male $(n=59)$ & Female $(n=41)$ & $P$ Value \\
\hline \multicolumn{5}{|l|}{ Type of disease } \\
\hline Acute symptomatic seizure & $94(94 \%)$ & $54(92 \%)$ & $40(98 \%)$ & 0.12 \\
\hline Neonatal epilepsy syndrome & $6(6 \%)$ & $5(8 \%)$ & $1(2 \%)$ & 0.08 \\
\hline \multicolumn{5}{|l|}{ Etiology of seizure } \\
\hline Neonatal encephalopathy and hypoxic-ischemic encephalopathy & $82(82 \%)$ & $49(83 \%)$ & $33(80 \%)$ & 0.82 \\
\hline Structural brain lesions & $6(6 \%)$ & $3(5 \%)$ & $3(7 \%)$ & 0.65 \\
\hline Intracranial hemorrhage & $3(3 \%)$ & $1(2 \%)$ & $2(5 \%)$ & 0.06 \\
\hline Central nervous system infections & $2(2 \%)$ & $2(3 \%)$ & $0(0 \%)$ & 0.09 \\
\hline Neonatal-onset epilepsy & $2(2 \%)$ & $1(2 \%)$ & $1(3 \%)$ & 0.16 \\
\hline Vaccine-associated & $2(2 \%)$ & $1(2 \%)$ & $1(3 \%)$ & 0.07 \\
\hline Hypocalcemia & $1(1 \%)$ & $0(0 \%)$ & $1(3 \%)$ & 0.84 \\
\hline Hypomagnesemia & $1(1 \%)$ & $1(2 \%)$ & $0(0 \%)$ & 0.34 \\
\hline Hyperglycemia & $1(1 \%)$ & $1(2 \%)$ & $1(0 \%)$ & 0.27 \\
\hline Family history of seizure & $8(8 \%)$ & $5(9 \%)$ & $3(6 \%)$ & 0.08 \\
\hline Previous history of seizure & $37(37 \%)$ & $24(40 \%)$ & $13(33 \%)$ & 0.21 \\
\hline
\end{tabular}


are not recognized clinically, and clinical features are poorly predictive of seizures in neonates with HIE. In a rigorous multicenter EEG monitoring study, about 50\% of neonates who received therapeutic hypothermia for HIE had EEG-confirmed seizures, and the most accurate predictor of seizures was a severely abnormal interictal EEG background during the first hour of recording. ${ }^{9}$ The diagnosis of neonatal encephalopathy and hypoxicischemic encephalopathy (HIE) can be difficult to make at times. Diagnostic criteria vary among societies, and established criteria may be too restrictive or failed to predict long-term neurologic sequelae. ${ }^{10-12} \mathrm{~A}$ study from Nepal reported seizure disorder as the commonest (33.4\%) cause of seizure in the studied patients followed by febrile seizures $(30.7 \%){ }^{13}$ indicating variety of seizure etiology in different communities. Hemorrhage (intracerebral, subarachnoid, intraventricular), ischemic stroke (arterial, watershed, and venous distributions), and congenital anomalies of the brain are the structural brain lesions associated with neonatal seizure. Hemorrhage and infarction may occur in isolation or may be the consequence of other etiologies such as CNS infection. Seizures are the most common sign of arterial ischemic stroke in newborn infants. ${ }^{14}$

Bacterial and viral infections of the CNS are important causes of seizures and other adverse sequelae. ${ }^{15}$ Prenatal infection is also a risk factor for seizure. Any neonate with suspected seizures should be considered to have a systemic and/or CNS infection until proven otherwise, and should have an immediate evaluation for infection. Often, empiric treatment is indicated until appropriate testing and cultures are completed. Potentially treatable metabolic etiologies include hypocalcemia, hypomagnesemia, and hypoglycemia. All newborns with suspected seizures should have a bedside glucose measurement, as well as laboratory testing for metabolic (electrolyte) disturbances. Typically, reversal of these abnormalities is sufficient to treat the acute symptomatic seizures, and anticonvulsant medications are not necessary. The results of the current study showed that hypoglycemia was not a common seizure etiology in the studied patients, although the commonest etiology of hypoglycemia after infancy in non-diabetic children is idiopathic ketotic hypoglycemia. ${ }^{1617}$ Glycogen storage disease and inborn errors of metabolism, hypopituitarism, ingestions, and sepsis are some hypoglycemia etiologies occurring during infancy and toddler years. ${ }^{18}$ Consistently, Chen et al ${ }^{19}$ in a study from Taiwan reported hypoglycemia as an infrequent cause of seizure.

A family history of seizure was observed only in $8 \%$ of the subjects that was the family history of febrile seizure in most of them (15/16) (data not shown). Hence, lack of a family history of seizure is fruitless in identifying the etiology or management of seizure. According to the results of a study, $8.2 \%$ of children experiencing first attack of seizure had a family history of seizure-related disorders. ${ }^{19}$

The prevalence of seizure etiology and type of seizure had no significant difference between males and females. However, the role of gender and influence of sex hormones on the occurrence of seizure and epilepsy as well as alterations of the endocrine system and sex hormones level by epileptic activities are indicated by clinical and experimental evidence. ${ }^{20}$

The study presented a clinico-etiological profile of seizure in Iranian newborns with neonatal seizure. The study had also some limitations. First, the cross sectional design of the study impeded any definitive diagnosis of seizure etiology in the patients. However, further studies with long-time follow-up design are suggested to investigate seizure relapse in patients and epileptiform activities. Second, imaging techniques were not employed in the study. Further studies may better elucidate seizure etiologies using such techniques.

In conclusion, the consequences of acute symptomatic seizures in neonates are determined mainly by the etiology of the seizures. Seizure burden and use of antiseizure drugs may also have some impact, but this has yet to be fully defined.

\section{Conflict of Interest Disclosures}

The authors declare that they have no conflict of interests.

\section{Ethical Statement}

This study complies with current ethical considerations and was approved by institutional ethics committee. Informed consent was obtained from the patients and the study protocol conforms to the ethical guidelines.

\section{References}

1. Agarwal M, Fox SM. Pediatric seizures. Emerg Med Clin North Am. 2013;31(3):733-54. doi: 10.1016/j.emc.2013.04.001.

2. Patterson JL, Carapetian SA, Hageman JR, Kelley KR. Febrile seizures. Pediatr Ann. 2013;42(12):249-54. doi: 10.3928/00904481-20131122-09.

3. Mizrahi EMWKSns. Epileptic syndromes in infancy, childhood and adolescence. 3rd ed. In: Roger J, Bureau M, Dravet $\mathrm{CH}$, eds. John Libbey; 2002. p. 15.

4. Friedman MJ, Sharieff GQ. Seizures in children. Pediatr Clin North Am. 2006;53(2):257-77. doi: 10.1016/j. pcl.2005.09.010.

5. Mizrahi EM. Neonatal Seizures. In: Panayiotopoulos CP, ed. Atlas of Epilepsies. London: Springer; 2010. p. 491-502. doi: 10.1007/978-1-84882-128-6_74.

6. Co JP, Elia M, Engel J Jr, Guerrini R, Mizrahi EM, Moshe $\mathrm{SL}$, et al. Proposal of an algorithm for diagnosis and treatment of neonatal seizures in developing countries. Epilepsia. 2007;48(6):1158-64. doi: 10.1111/j.15281167.2007.01008.x.

7. Glass HC. Neonatal seizures: advances in mechanisms and management. Clin Perinatol. 2014;41(1):177-90. doi: 10.1016/j.clp.2013.10.004.

8. Leung AK, Robson WL. Febrile seizures. J Pediatr Health Care. 2007;21(4):250-5. doi: 10.1016/j.pedhc.2006.10.006.

9. Rothman SM, Glass HC, Chang T, Sullivan JE, Bonifacio $\mathrm{SL}$, Shellhaas RA. Risk factors for EEG seizures in neonates 
treated with hypothermia: a multicenter cohort studyAuthor Response. Neurology. 2014;83(19):1773-4. doi: 10.1212/01. wnl.0000456637.05253.24.

10. Nelson KB, Leviton A. How much of neonatal encephalopathy is due to birth asphyxia? Am J Dis Child. 1991;145(11):132531. doi: 10.1001/archpedi.1991.02160110117034.

11. Gaffney $G$, Flavell V, Johnson A, Squier M, Sellers S. Cerebral palsy and neonatal encephalopathy. Arch Dis Child Fetal Neonatal Ed. 1994;70(3):F195-200. doi: 10.1136/ fn.70.3.f195.

12. Leviton A, Nelson KB. Problems with definitions and classifications of newborn encephalopathy. Pediatr Neurol. 1992;8(2):85-90. doi: 10.1016/0887-8994(92)90026-u.

13. Adhikari S, Sathian B, Koirala DP, Rao KS. Profile of children admitted with seizures in a tertiary care hospital of Western Nepal. BMC Pediatr. 2013;13:43. doi: 10.1186/1471-243113-43.

14. Kirton A, Deveber G. Life after perinatal stroke. Stroke. 2013;44(11):3265-71. doi: 10.1161/strokeaha.113.000739.
15. Verboon-Maciolek MA, Truttmann AC, Groenendaal F, Skranes J, Dollner H, Hunt RW, et al. Development of cystic periventricular leukomalacia in newborn infants after rotavirus infection. J Pediatr. 2012;160(1):165-8.e1. doi: 10.1016/j.jpeds.2011.08.053.

16. Daly LP, Osterhoudt KC, Weinzimer SA. Presenting features of idiopathic ketotic hypoglycemia. J Emerg Med. 2003;25(1):39-43. doi: 10.1016/s0736-4679(03)00100-8.

17. Freedman BD, Hughan K, Garibaldi L. Hypoglycemic seizure. Clin Pediatr (Phila). 2010;49(11):1078-80. doi: 10.1177/0009922809337537.

18. Hoe FM. Hypoglycemia in infants and children. Adv Pediatr. 2008;55:367-84.

19. Chen $\mathrm{CY}$, Chang YJ, Wu HP. New-onset seizures in pediatric emergency. Pediatr Neonatol. 2010;51(2):103-11. doi: 10.1016/s1875-9572(10)60019-8.

20. Velíšková J, Desantis KA. Sex and hormonal influences on seizures and epilepsy. Horm Behav. 2013;63(2):267-77. doi: 10.1016/j.yhbeh.2012.03.018. 